\title{
Challenges in Postprocessing of Hybrid Manufacturing of Magnet Polymer Composites to Enhance the Energy Product of NdFeB Bonded Magnets
}

ISSN: 2576-8840

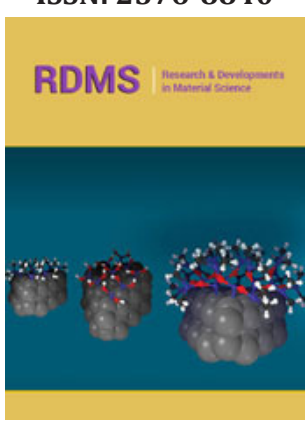

*Corresponding author: Tej Nath Lamichhane and M Parans Paranthaman, Chemical Science Division, Oak Ridge National Laboratory, Oak Ridge, TN 37831, USA Emails: lamichhanetn@ornl.gov paranthamanm@ornl.gov

Submission: 眥 November 17, 2021

Published: 制 November 24, 2021

Volume 16 - Issue 2

How to cite this article: Tej Nath Lamichhane, Kaustubh Mungale, Haobo Wang, Kinjal Gandha, M Parans Paranthaman, et al., Challenges in Postprocessing of Hybrid Manufacturing of Magnet Polymer Composites to Enhance the Energy Product of NdFeB Bonded Magnets. Res Dev Material Sci. 16(2). RDMS.000882. 2021.

DOI: 10.31031/RDMS.2021.16.000882

Copyright@ Tej Nath Lamichhane. This article is distributed under the terms of the Creative Commons Attribution 4.0 International License, which permits unrestricted use and redistribution provided that the original author and source are credited.

\author{
Tej Nath Lamichhane ${ }^{1 *}$, Kaustubh Mungale ${ }^{2,3,4}$, Haobo Wang ${ }^{1,5}$, Kinjal Gandha ${ }^{6}$, \\ Xubo Liu ${ }^{6}$, Ikenna C Nlebedim ${ }^{6}$, Zhang Gai $^{7}$, Uday Kumar Vaidya ${ }^{2,3,4}$ and $\mathrm{M}$ \\ Parans Paranthaman ${ }^{1 * *}$ \\ ${ }^{1}$ Chemical Science Division, Oak Ridge National Laboratory, Oak Ridge, TN 37831, USA \\ ${ }^{2}$ Tickle College of Engineering, The University of Tennessee, Middle Drive, Knoxville, TN \\ 37996, USA
}

${ }^{3}$ Manufacturing Sciences Division, Oak Ridge National Laboratory, 2350 Cherahala Blvd, Knoxville, TN 37932, USA

${ }^{4}$ Institute for Advanced Composites Manufacturing Innovation, 2360 Cherahala Blvd, Knoxville, TN 37932, USA

${ }^{5}$ The Bredesen Center, The University of Tennessee, Knoxville, TN 37996, USA

${ }^{6}$ Critical Materials Institute, Ames Laboratory, Ames, IA 50011, USA

${ }^{7}$ Center for Nanophase Materials Sciences Division, Oak Ridge National Laboratory, Oak Ridge, TN 37831, USA

\begin{abstract}
Bonded permanent magnets need to process magnetic filler particles and binder together which is a form of hybrid or composite manufacturing that needs to simultaneously process multimaterials. Polymer bonding of the permanent magnets decreases the energy product of the bonded magnets which varies with square of loading fraction of magnetic materials. However, due to high viscosity of bonding polymer prohibits loading higher than 80 volume \% of magnetic materials in most efficient loading process such as compression molding. To further increase the loading of magnetic fraction in the bonded permanent magnets, it is expected to be beneficial to introduce a fraction of nonreacting thermally sacrificial polymer along with permanent bonding polymer in the least permissible of about 20 volume $\%$ of the polymer. This process of first adding the sacrificial polymer for higher processability for composites and later removing it via post processing such as heat treatment provides a simple example of inclusion of both the additive and the subtractive stages of hybrid manufacturing. These hybrid composite magnets can be heat treated to remove the sacrificial polymer to further increase the density and energy product which might require to implement hot isostatic pressing for compaction. Although the identification of such ideal polymer requires an extensive polymer chemistry research, we have compression molded several permanent bonding polymers such as nylon, polycarbonate (PC) and polyphenyl sulfide (PPS) and potential sacrificial polymer such as polyoxymethylene (POM), polystyrene (PS), and Acrylonitrile Butadiene Styrene (ABS) and heat treated to remove the sacrificial polymers. It is discovered that oxygen free low temperature pyrolizable polymers could be the potential candidates for increasing the magnet particles loading for achieving high energy products.
\end{abstract}

\section{Introduction}

Hybrid manufacturing process encompasses combination of multiple processing techniques as well as simultaneous processing of multimaterials to improve the overall quality of the process and products [1]. Permanent magnets energy product boosting is a critical manufacturing challenge both in sintered magnets and bonded magnets. Most powerful grade 
of magnets are traditionally sintered magnets which require several conventional processing steps such as powder metallurgy [2], strip casting [3], and complex thermal treatment cycles. These complex manufacturing steps makes the permanent magnets coprocessing with other electrical machines components such as magnet holder rotor core very complicated if not impossible. Broadly speaking, the electrical machines in this article refers to motors, generators, power electronics and additional devices that incorporates soft magnets, permanent magnets, electrical conductors, cooling channels and sensors. In bonded magnets, there are several wellestablished techniques such as compression molding, injection molding, calendaring and extrusion [4] which can be categorized as hybrid or semihybrid manufacturing technique since the magnetic filers need to be blended with binders and coprocessed to manufacture the magnets. The extrusion based bonded magnets manufacturing technique is emerging as additive manufacturing also known as 3D printing technique such as fused deposition modeling where the magnets composites can be printed in complex shapes $[5,6]$. Bonded magnets are very important to reduce the weight of an electrical machine, eddy current loss, increase the flexural strength and complex shapes and sizes moldability [7].

There are very few hybrid magnets manufactured literature reports mainly in form of 3D printing of two types of powders and binders. Yang et al. 3D printed the mixture of anisotropic NdFeB and $\mathrm{SrFe}_{12} \mathrm{O}_{19}$ powder using epoxy extrusion technique and magnet density of 3.78-3.64g/cc depending upon content of mixed ferrite powder [8]. Similarly, Gandha et al. Used the fused deposition modeling based big area additive manufacturing of anisotropic $\mathrm{NdFeB}$ and Sm-Fe-N powder in nylon binder and heat treated in external magnetic field to generate up to 11 MGOe energy product [9]. There are not many initiatives of removal of some nonmagnetic fragments of binder from post processing such as heat treatment or chemical removal of a component and consolidation at the end of hot isostatic pressing is not pursued as an avenue to enhance the energy product of the fabricated magnets. Rather the energy product of the bonded magnets is greatly reduced due to inclusion of nonmagnetic binders where energy product varies as the square of the filling fraction [10]. In the current most efficient filling method, compression molding can process up to 80 volume \% [11] of magnetic filer in the commercial binders, in general permanent, such as nylon, polyphenylene sulfide (PPS) and polycarbonates (PC). In this article the permanent polymers refers to commercial, high temperature melting magnetic binders such as nylon, PPS and PC. An 80 volume \% magnetic fillers packing cannot provide more than $64 \%$ of the energy product of the starting magnetic powder which could be up to 25 MGOe for the 40 MGOe anisotropic precursor powders. However, to further increase the energy product of the bonded magnets, there is need to develop a new method of increasing the volume fraction of the magnetic fillers which is already optimized for most of the commercial magnetic binders.

Hybrid manufacturing with integrated multimaterials processing of additive manufacturing technologies and subtractive modes of parts accessing, surface treatments, tolerance maintenance and postprocessing capabilities would be the next steps towards the production of the next generation of electrical machines [12]. Such hybrid production units would be very essential for the onsite production and installation of large high performance electrical machines such as wind turbines. Since the high-performance electrical machines needs high energy product sintered magnets, it is essential to increase the energy product of the bonded magnets to make them more applicable. Large electrical machines and their components are inherently complicated to manufacture due to their component's wise complex materials composition as well as gigantic structures. Bonded magnets can help in significant weight reduction in gigantic electrical machines without much compromise in performance since they could significantly reduce the eddy current loss $[7,13]$. Hybrid manufacturing of electrical machines is essential for the next generation of electrical machines which includes onsite manufacturing, installation and boosting the overall performance [12]. Recently General Electric and Company has announced the additive manufacturing of nacelle and additional large metallic components of wind turbines which also includes sand/ceramics ink jetting for the mold's fabrication [14]. This trend indicates that the future large electrical machines manufacturing techniques will evolve toward hybrid manufacturing method to increase the ease of design and productivity which must come up with fundamental challenges of simultaneous processing of materials for several components [15]. One of the biggest challenges of a single-attemptbuilt electrical machines would be simultaneous processing of soft magnetic materials for electrical cores, permanent magnets, winding electrical conductors and insulating materials due to their very dissimilar materials processing techniques and thermal cycles. Being the key component of electrical machines, investigation about the possibility of coprocessing of permanent magnets in hybrid manufacturing of electrical machines is essential. Due to very high temperature thermal processing cycles of soft magnets and electric conductors in comparison to permanent magnets, a low temperature thermal processing at the final stage of the hybrid manufacturing process is essential for permanent magnets. An intuitive approach could be to come up with stepwise processing of multimaterials components for the electrical machines such as casting of soft laminates separated by thin, soft magnetic insulator laminates [12], casting/printing of unconventional conducting cables [16-18], cooling channels followed by permanent magnets to achieve the single built electrical machine [19]. Among these steps, permanent magnets are the most expensive part of the electrical machines and cannot be easily casted in complex shapes 
and large sizes and properly magnetized in the desired direction. This is due to the requirement of complex thermal cycles for the magnets microstructure refinement to achieve their monomagnetic domain sizes, their alignment and magnetization to achieve the permanency specially in fully sintered magnets. In such a scenario, bonded permanent magnets still could be processed via either inkjetting of soft magnetic grain boundary phase laden ink to bond the preoptimized monodomain anisotropic magnetic particles or extruding the magnet polymer composite in the designated slots for the permanent magnets. However, low eddy current loss is a very important factor for achieving higher efficiency in electrical machines and requires thin laminates of soft magnetic materials. Similarly in bonded permanent magnets, the eddy current could be greatly reduced since the polymer binding greatly increases the electrical resistance and loss fraction of magnetization [7]. One of the options could be to compress molding large permanent magnets sections in a soft steel enclosure, heat treat and enhance the energy product via partial removal of nonmagnetic mostly sacrificial binder and high temperature isostatic pressing, and hermetically seal and magnetize them before they are installed in the ceramics casted slots of electrical rotors.

To exceed the current loading fraction of magnetic particles and reap the benefits of lower eddy current in bonded magnets, we investigated the opportunities and challenges of hybrid manufacturing of bonded magnets with permanent and sacrificial binder polymers [20] with maximum particles loading (e.g., 80 volume $\%$ of $\mathrm{NdFeB}$ ) using compression molding and post processing. The key task was to fabricate the bonded $\mathrm{NdFeB}$ magnets with two types of binder and heat treat to remove the low temperature melting "hereby sacrificial polymer" to increase the overall density of the bonded magnets. To successfully execute this idea, the biggest challenge starts from identification of possible sacrificial binder that does not react with permanent binder polymer while heat treating and does not degrade the magnet properties during low temperature pyrolysis. We selected the easily available low melting polymers both oxygenated and oxygen free polymers as sacrificial binders, compressed molded with permanent binders such as nylon, PPS and PC and postprocessed via heat treatment under Ar atmosphere. The preliminary study revealed that oxygen free low temperature pyrolyzable polymers that do not react with permanent binders and do not degrade the magnetic particles would be the ideal candidates to be discovered in future. An alternative route for the sacrificial binder removal could be chemical removal [21], which could be further accelerated via selective catalytic decomposition [22] of sacrificial polymer are the area to be investigated in future.

\section{Method}

As permanent binders, relatively higher melting temperature with well-established processing techniques such as nylons, PPS, and PC were selected. As potential sacrificial polymers low melting temperature polymers (polyoxymethylene (POM), polystyrene (PS) and acrylonitrile butadiene styrene (ABS) were selected (chemical formula and melting temperatures are reported in Table 1). Anisotropic Magnequench magnet powders were used for the compression molding. The process of the compression molding of the composite is reported elsewhere [10]. Heat treatment of the composites to remove the sacrificial polymers were carried out under the Ar flow environment both with and without magnetic field up to 10 hours. The magnetic properties of the samples were measured using Quantum Design Dynacool magnetometer with a magnetic field of up to $\mu_{0} H=9 T$.

Table 1: Description of trailed polymers. Top three are permanent and lower three are considered sacrificial.

\begin{tabular}{|c|c|c|c|}
\hline Polymers & Formula & Melting Temperature ( $\left.{ }^{\circ} \mathrm{C}\right)$ & Remarks \\
\hline Polyphenylene sulphide & & \\
\hline Nylon & & 280 \\
\hline Polycarbonate & \\
\hline
\end{tabular}




\begin{tabular}{|c|c|c|c|}
\hline Polyoxymethylene & & & \\
& & & \\
& & & \\
\hline
\end{tabular}

\section{Results and Discussion}

In case of sacrificial polymer with oxygen, the magnetic coercivities of the heat-treated samples were almost completely lost. As a representative result, PC bonded magnet compounded with POM showed significant loss of coercivity after heat treatment at $600{ }^{\circ} \mathrm{C}$ to remove all the weight of sacrificial polymer POM compared with as-compounded sample (Figure 1). Additionally, the mechanical properties of the sample were degraded significantly. This outcome suggests that the sacrificial polymer removal should be executed in relatively low temperature. Another parameter is the time of heat treatment so that coercivity of the magnet is also not degraded in addition to mechanical properties. Ultimately, to achieve the dense magnet, hot isostatic pressing will be essential to increase the overall density of the magnet [23].

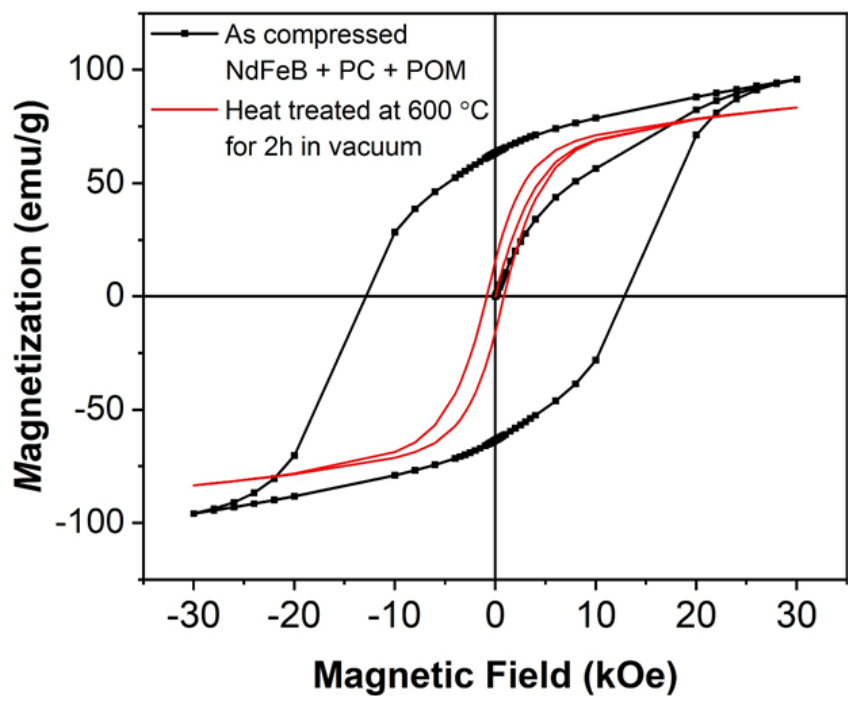

Figure 1: Room temperature magnetization of as compressed molded and $600{ }^{\circ} \mathrm{C} 2 \mathrm{~h}$ heat treated $\mathrm{NdFeB}$, polycarbonate and polyoxymethylene bonded magnets until the weight loss percentage corresponding of sacrificial polymer.

Figure 2 shows the magnetic properties of the $\mathrm{NdFeB}$, nylon and ABS compression molded magnets. Figure 2(a) shows the room temperature magnetization of as printed and heat-treated samples in Ar atmosphere for $10 \mathrm{~h}$ which shows a significant $(11.5 \%)$ decrease in coercivity. Figure 2(b) shows the room temperature magnetization for the same batch heat treated in presence of the $1 \mathrm{~T}$ magnetic field at $300{ }^{\circ} \mathrm{C}$ for $2 \mathrm{~h}, 5 \mathrm{~h}$ and $10 \mathrm{~h}$ dwell times. Corresponding coercivity, \% mass loss of the samples and saturation magnetization are shown in Table 2. In comparison to coercivity, the saturation magnetization loss is negligible. As expected, preserving the coercivity is a crucial challenge in hybrid magnet manufacturing as it is for generating in sintering techniques. The most important outcome of the Nylon/ABS composite is that although nylon has oxygen in it, it does not degrade the magnets. There is almost comparable coercivity in the as printed sample for the sample which was heat treated under Ar flow for about $5 \mathrm{~h}$ as shown in Figure 2(b). The coercivity is slightly decreased in $10 \mathrm{~h}$ heat treatment time. 

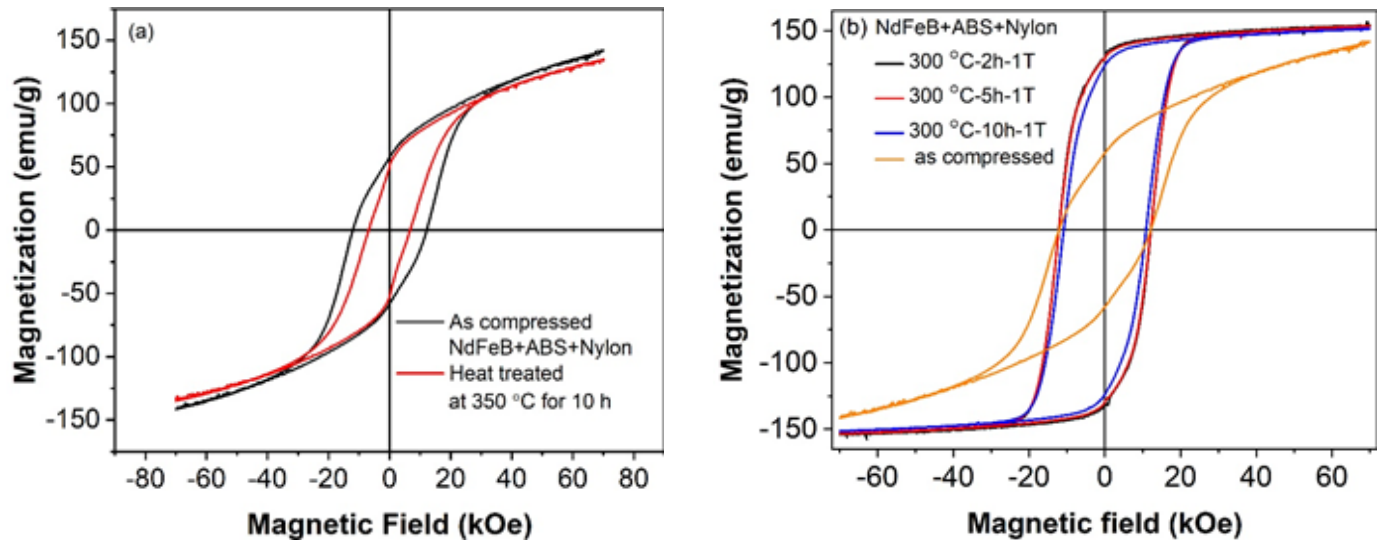

Figure 2: Comparison of room temperature magnetization of as compressed molded and heat treated $\mathrm{NdFeB+}$ ABS + Nylon composite (a) without applied field (b) with applied field.

Table 2: Measurement of coercivities, \% mass loss and saturation magnetization in the heat-treated ABS+Nylon NdFeB magnet composite.

\begin{tabular}{|c|c|c|c|}
\hline Dataset ID & Coercivity (k0e) & Mass loss \% of the Sample & Saturation Magnetization (emu/g) \\
\hline ABS+Nylon-300 ${ }^{\circ} \mathrm{C}-2 \mathrm{~h}-1 \mathrm{~T}$ & 12.2 & 0.32 & 154.0 \\
\hline ABS+Nylon-300 ${ }^{\circ} \mathrm{C}-5 \mathrm{~h}-1 \mathrm{~T}$ & 12.2 & 0.61 & 153.4 \\
\hline ABS+Nylon-300 ${ }^{\circ} \mathrm{C}-10 \mathrm{~h}-1 \mathrm{~T}$ & 10.8 & 0.81 & 151.4 \\
\hline ABS+Nylon as compounded & 12.2 & - & 141 \\
\hline
\end{tabular}

Figure 3 shows the comparison of magnetic hysteresis loops of as compounded and heat treated NdFeB/PPS/ABS composite samples without applied magnetic field in Figure 3(a) and with applied magnetic field in Figure 3(b). The coercivities, mass loss
$\%$ and saturation magnetizations are presented in Table 3. The coercivity of the heat treated NdFeB PPS/ABS composite samples are drastically decreasing despite no oxygen both in PPS and ABS.
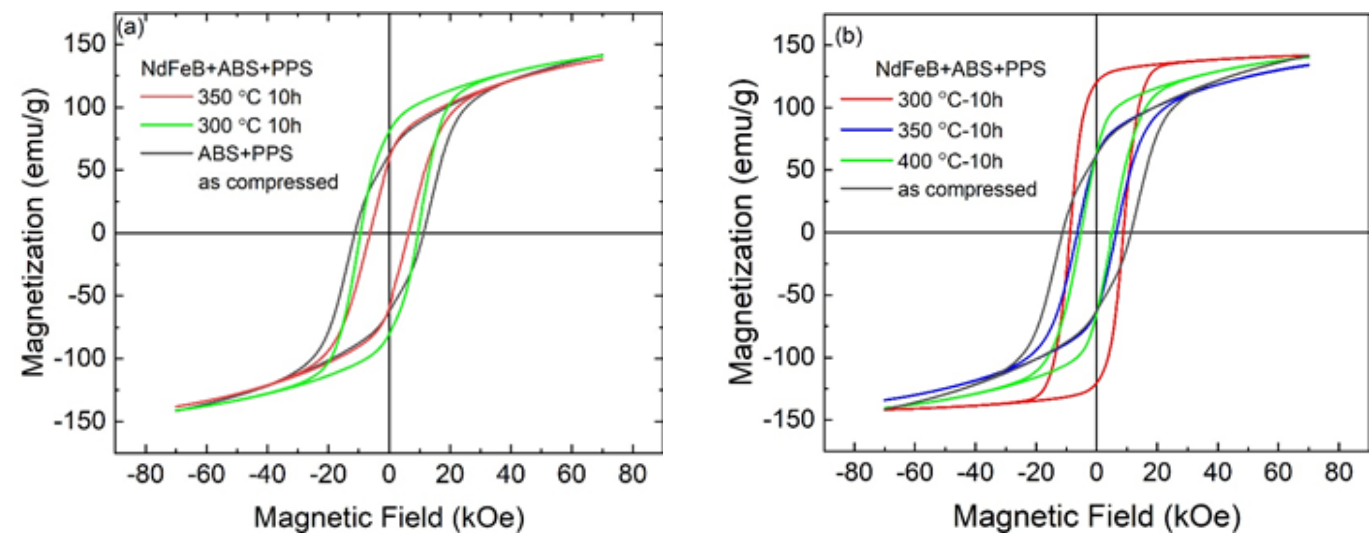

Figure 3: Comparison of room temperature magnetization of as compressed molded and heat treated $\mathrm{NdFeB}+\mathrm{PPS}+\mathrm{ABS}$ composite (a) without field (b) with applied field.

Table 3: Measurement of coercivities, \% mass loss and saturation magnetization in the heat-treated ABS/PPS NdFeB magnet composite.

\begin{tabular}{|c|c|c|c|}
\hline Dataset ID & Coercivity (k0e) & Mass loss \% of the Sample & Saturation Magnetization (emu/g) \\
\hline ABS + PPS as compounded & 11.4 & - & 141.6 \\
\hline ABS + PPS $-300{ }^{\circ} \mathrm{C}-1 \mathrm{~T}$ & 9.4 & 0.4 & 141.9 \\
\hline ABS + PPS $-350^{\circ} \mathrm{C}-1 \mathrm{~T}$ & 6.19 & 0.5 & 138.2 \\
\hline $\mathrm{ABS}+\mathrm{PPS}-400^{\circ} \mathrm{C}-1 \mathrm{~T}$ & 5.1 & 1.48 & 141 \\
\hline
\end{tabular}


From the experimental evidence of compression molding of $\mathrm{NdFeB}$, low melting temperature and oxygen-free sacrificial polymers like ABS is a promising candidate. In case of oxygenated and oxygen free permanent polymer, it is not clear whether oxygen from those permanent polymers is detrimental below melting temperature of permanent polymer or not. In fact, the role of oxygen constituent in polymer binders for bonded magnets was not previously reported. As the hybrid manufacturing is getting unprecedented attention and permanent magnet is the key component of the electrical machines, it is crucial to find a proper way of coprocessing of permanent magnets designing as well as enhancing the energy produce after initial manufacturing. In this regards, low temperature processing techniques such as compressing molding, fused deposition modeling, binder jetting etc. can be combined to already casted/printed soft magnetic rotor slots without altering the mechanical and magnetic properties of the soft magnetic materials. As the post processing of ABS/Nylon shows very encouraging result although we just tested few pairs of polymers. As this research will progress, it needs to identify appropriate pair of polymers to remove a sacrificial polymer without sacrificing the magnetic properties. The future area to focus could be identifying low temperature, low heat treatment time and easy hot isostatic pressing technique to increase the density of the permanent magnets.

\section{Conclusion}

The preliminary concept of hybrid manufacturing of $\mathrm{NdFeB}$ bonded magnets various permanent commercial binders such as PC, nylon, and PPS mixed with various low melting polymers with and without constituent oxygen. The Nylon /ABS binder pairs preserve the magnetic properties almost intact among the tested pairs up to $10 \mathrm{~h}$ heat-treatment under inert atmosphere although much high temperature heat treatment is essential to completely remove the sacrificial components. These preliminary results highlight the area to focus on identifying low temperature, oxygen free pyrolyzable sacrificial polymer along with easily processable permanent binder such as nylon.

\section{Acknowledgement}

This research was supported by the U.S. Department of Energy, Office of Energy Efficiency and Renewable Energy, Advanced Manufacturing Office. This manuscript has been authored by UT-Battelle, LLC, under contract DE-AC05-000R22725 with the US Department of Energy (DOE). The US government retains and the publisher, by accepting the article for publication, acknowledges that the US government retains a nonexclusive, paid-up, irrevocable, worldwide license to publish or reproduce the published form of this manuscript, or allow others to do so, for US government purposes. DOE will provide public access to these results of federally sponsored research in accordance with the DOE Public Access Plan (http://energy.gov/downloads/doe-public-access-plan).

\section{References}

1. Zhu Z, Dhokia VG, Nassehi A, Newman ST (2013) A review of hybrid manufacturing processes - state of the art and future perspectives. Int J Comput Integr Manuf 26(7): 596-615.

2. Constantinides S. Manufacture of modern permanent magnet materials. p. 12.

3. Kaneko Y, Kuniyoshi F, Ishigaki N (2021) Proven technologies on highperformance Nd-Fe-B sintered magnets. J Alloys Compd 408-412: 13441349.

4. Staff E (2015) Bonded magnets: A versatile class of permanent magnets. Magnetics Magazine.

5. Li L, Tirado A, Nlebedim IC, et al. (2016) Big area additive manufacturing of high performance bonded NdFeB magnets. Sci Rep 6(1): 36212.

6. Li L, Post B, Kunc V, Elliott AM, Paranthaman MP (2017) Additive manufacturing of near-net-shape bonded magnets: Prospects and challenges. Scr Mater 135: 100-104.

7. Paranthaman MP, Yildirim V, Lamichhane TN, et al. (2020) Additive manufacturing of isotropic NdFeB PPS bonded permanent magnets. Materials 13(15): 3319

8. Yang F, Zhang X, Guo Z, Ye S, Sui Y, et al. (2019) 3D printing of NdFeB bonded magnets with SrFe12019 addition. J Alloys Compd 779: 900907.

9. Gandha K, Li L, Nlebedim IC, et al. (2018) Additive manufacturing of anisotropic hybrid NdFeB-SmFeN nylon composite bonded magnets. J Magn Magn Mater 467: 8-13.

10. Mungale K, Lamichhane TN, Wang H, Sales BC, Paranthaman MP, et al. Compression molding of anisotropic $\mathrm{NdFeB}$ bonded magnets in a polycarbonate matrix. Materialia 19: 101167

11. Ormerod J, Constantinides S (1997) Bonded permanent magnets: Current status and future opportunities (invited). J Appl Phys 81(8): 4816-4820.

12. Lamichhane TN, Sethuraman L, Dalagan A, Wang H, Keller J, et al. (2020) Additive manufacturing of soft magnets for electrical machines-a review. Mater Today Phys 15: 100255.

13. Li L, Jones K, Sales B, Pries JL, Nlebedim IC, et al. (2018) Fabrication of highly dense isotropic Nd-Fe-B nylon bonded magnets via extrusionbased additive manufacturing. Addit Manuf 21: 495-500.

14. (2021) GE \& Co to develop world's largest 3D printer for offshore wind application. Offshore Wind.

15. Hayes AC, Whiting GL (2021) Reducing the structural mass of large direct drive wind turbine generators through triply periodic minimal surfaces enabled by hybrid additive manufacturing. Clean Technol 3(1): 227-242.

16. Yan Y, Ding C, Ngo KDT, Mei Y, Lu GQ (2016) Additive manufacturing of planar inductor for power electronics applications. In: 2016 international symposium on 3D power electronics integration and manufacturing (3D-PEIM). 2016: 1-16.

17. Ding C, Lu S, Liu L, Ngo KDT, Lu GQ (2021) Additive manufacturing of hetero-magnetic coupled inductors. IEEE Trans Compon Packag Manuf Technol 11(6): 1028-1034. 
18. Additive drives: 3D printed windings for electric cars. 3DPrint.com.

19. Wu F, EL-Refaie AM (2020) Toward additively manufactured electrical machines: Opportunities and challenges. IEEE Trans Ind Appl 56(2): 1306-1320.

20. Uzunlar E, Schwartz J, Phillips O, Kohl PA (2016) Decomposable and template polymers: Fundamentals and applications. J Electron Packag 138(2): 020802.
21. Linder V, Gates BD, Ryan D, Parviz BA, Whitesides GM (2005) Watersoluble sacrificial layers for surface micromachining. Small Weinh Bergstr Ger 1(7): 730-736.

22. Liu L, Zachariah MR, Stoliarov SI, Li J (2015) Enhanced thermal decomposition kinetics of poly(lactic acid) sacrificial polymer catalyzed by metal oxide nanoparticles. RSC Adv 5(123): 101745-101750.

23. Sagawa M, Nagata H, Watanabe T, Itatani O (1997) Rubber Isostatic Pressing (RIP) for ferrite magnets. J Phys IV Proc 07(C1): C1-307-C1-308.

For possible submissions Click below: 